\title{
Characterization of the mutual influence of Ion Cyclotron and Lower Hybrid Range of frequencies systems on EAST
}

\author{
Guillaume Urbanczyk ${ }^{1,2,3,4,{ }^{*}}$, Xinjun Zhang ${ }^{1}$, Chengming Qin ${ }^{1}$, Yanping Zhao ${ }^{1}$, Tao Zhang ${ }^{1}$, Ling Zhang ${ }^{1}$, Jiangang Li ${ }^{1}$,

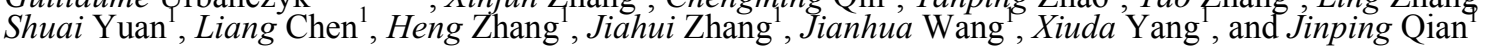 \\ ${ }^{1}$ Institute of Plasma Physics, Chinese Academy of Sciences, Hefei 230031, People'sRepublic of China \\ ${ }^{2}$ Université de Lorraine-CNRS UMR 7198 BP 70239 FST_IJL F-54506 Vandoeuvre \\ ${ }^{3}$ University of Science and Technology of China, Hefei 230026, People's Republic of China \\ ${ }^{4}$ CEA, IRFM, F-13108 Saint Paul-lez-Durance, France
}

\begin{abstract}
Waves in the Ion Cyclotron (ICRF) and Lower Hybrid (LH) Range of Frequencies are efficient techniques respectively to heat the plasma and drive current. Main difficulties come from a trade-off between good RF coupling and acceptable level of impurities release. The mutual influence of both systems makes such equilibrium often hard to reach [1]. In order to investigate those interactions based on ScrapeOff Layer (SOL) plasma parameters, a new reciprocating probe was designed allying a three tips Langmuir probe with an emissive wire. The emissive filament provides a precise measure of plasma potential [2], which can be used to calibrate Langmuir probe's results. This paper reports on experimental results obtained on EAST, where there are two ICRF antennas and two LH launchers. Among others diagnostics, the new reciprocating probe enabled to evidence the deleterious influence of ICRF power on LHWs coupling in Lmode plasmas. In areas connected with an active ICRF antenna, SOL potentials increase while densities tend to decrease, respectively enhancing impurities release and deteriorating LHWs coupling. This phenomenon has mostly been attributed to RF sheath; the one that forms on top of Plasma Facing Components (PFCs) and causes ExB density convections [3]. From those experiments it seems ICRF has a strong influence on magnetically connected areas, both in the near field - influencing ICRF waves coupling - and in farther locations such as in front of LH grills. Moreover, influence of ICRF on LH system was observed both in $\mathrm{L}$ and $\mathrm{H}$ modes. Those results are consistent with RF sheath rectification process. Concerning the influence of LHWs on ICRF coupling, nothing was observed in L-mode. Besides during Hmode experiments, LHWs have been identified as having a mitigating effect on ELMs [4], which on average lowers the pedestal, increasing edge densities to the profit of ICRF waves coupling.
\end{abstract}

\section{Introduction}

Lower Hybrid Current Drive (LHCD) consists in launching waves at a frequency lying in the $\mathrm{GHz}$ range of frequencies. Excited by klystrons, waves are transported into waveguides and launched in the plasma from a grill. Since LH Waves (LHWs) are evanescent at too low densities (bellow $7.10^{17} \mathrm{~m}^{-3}$ at $4.6 \mathrm{GHz}$ ), gas puff is often used to increase electron densities locally in front of the grill to improve coupling efficiency. Thanks to their large parallel electric field, LHWs have the interesting property of damping very efficiently at high parallel phase velocities to the electron thermal speed. They are consequently able to drive off-axis current, allowing good control of plasma current profile, enabling to build internal transport barriers and access attractive steady-state operations with high bootstrap current. On ITER, LHCD system should enable to reach more than $70 \%$ of bootstrap current, with high $\beta_{\mathrm{N}}(\sim 3)$ and good confinement $\left(\mathrm{H}_{\mathrm{ITER}-89} \sim 2.5\right)$ [5].

Ion Cyclotron Range of Frequencies (ICRF) goes from 10 to $100 \mathrm{MHz}$. ICRF waves are mostly being used for heating plasmas on tokamaks. Unlike LHWs, ICRF waves are excited from an antenna composed of current straps as close as possible to the plasma. ICRF waves are also evanescent at low densities and must tunnel through a thin evanescent layer before reaching higher density plasma where they can propagate normally until reaching a resonance layer and essentially exchange their energy with ions before being spread to the whole plasma by collisions. Unfortunately, single-pass absorption and coupling efficiency are never perfect, so that waves undergo several reflections in the Scrape-Off Layer (SOL), enhancing dramatically interactions with Plasma Facing Components (PFCs). Those interactions are generally the result of RF sheaths which characterization from a global point of view can be done through an increase of impurities production and consequently an increase in radiations. From a local point of view, ExB convections induce strong modifications on density profiles at the edge, often degrading both ICRF and LH waves coupling efficiencies.

By presenting experiments recently conducted on Experimental Advanced Superconducting Tokamak, we aimed at characterizing interaction processes during ICRH and LH operations. A brief overview of EAST and the position of objects and diagnostics of interests for this study are given in section 2. Further details on the design of a newly designed reciprocating probe which enabled to collect key information 
for this study are provided in section 3. Finally experimental results are addressed in section 4.

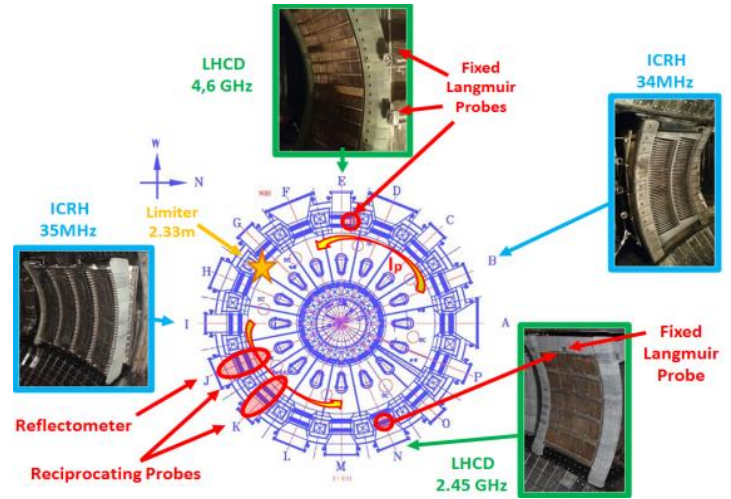

Fig. 1. Plan of EAST with each diagnostic of interest and its toroidal position

\section{EAST presentation}

Two ICRF antennas and two LH grills are available on EAST. In order to minimize interactions between each launcher, their positions were changed after the 2014 campaign to maximize distances between them (Fig. 1.). B and I ports ICRF antennas such as $\mathrm{E}$ and $\mathrm{N}$ ports $\mathrm{LH}$ grills are almost diametrically opposite in the torus to minimize mutual influences. While 6MW power is available on each ICRF antenna roughly working at same frequencies ( 34 and $35 \mathrm{MHz}$ ), each $\mathrm{LH}$ grill has different power, frequency, and wave number range; respectively $4 \mathrm{MW}$ for $\mathrm{N}$-port at $2.45 \mathrm{GHz}$ with $2<\mathrm{n} \|<3$, and $6 \mathrm{MW}$ for E-port at $4.6 \mathrm{GHz}$ with $1.8<\mathrm{n} \|<2.5$. Langmuir probes were also fixed on each LH grill, respectively on top of the N-port and on the right side of the E-port. Reciprocating probes can be inserted from $\mathrm{J}$ and $\mathrm{K}$ ports, with an $\mathrm{X}$-mode reflectometer in J-port [7] providing density profiles potentially useful to benchmark both probes results. However, the reflectometer's frequency range (from 4 to $6 \mathrm{GHz}$ ) is not appropriate to measurements in the SOL; it can only provide good results at densities above $10^{18} \mathrm{~m}^{-3}$. On the C-port, an Extreme UV spectrometer enables measurement of high-Z impurities in the plasma core [7].

\section{Reciprocating Emissive and Langmuir probe}

This probe (Fig. 2.) was specifically designed for the study of ICRF waves influence on the SOL. It consists in a combination of a $2 \mathrm{~cm}$ tungsten wire of $150 \mu \mathrm{m}$ diameter, doped with thorium

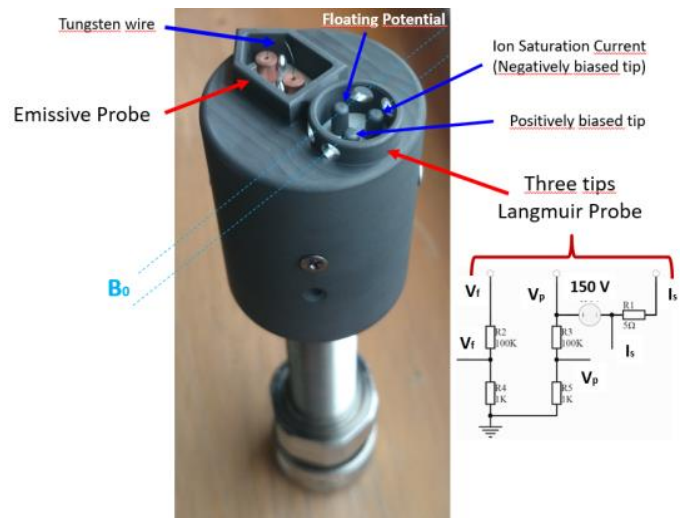

Fig. 2. Reciprocating probe allying three tips Langmuir and emissive techniques

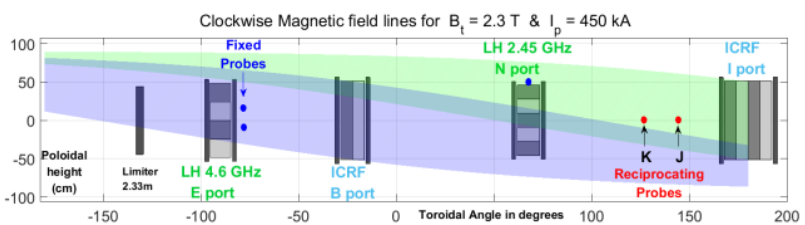

Fig. 3. Magnetic connection lines for clockwise magnetic field

to boost its emissive properties and a three tips Langmuir probes. Because emissive probe is hot, it enables to measure the potential of the plasma with much better precision than cold Langmuir probes. The idea is then to use emissive wire floating potential instead of Langmuir tips one, to calculate more precise values of plasma potential, electrons temperature and density. Reciprocations are fully controlled shot by shot from the main control room. Plunges timing, deepness, stationary laps inside the plasma are all set before each shot. Installation of the probe can conveniently be done in a small chamber which can be isolated from tokamak vacuum. Once the probe is ready, vacuum is restored after about two days pumping, and the probe can enter in the tokamak. This was particularly useful for experiments with the emissive probe, because unlike in machines with short discharges, EAST provides several seconds plasmas so that tungsten filament rapidly breaks after few plunges. Probes can be inserted into the middle plane of $J$ and $\mathrm{K}$ ports, both well connected magnetically to I-port and not connected to B-port ICRF antennas (Fig. 3.).

\section{Experimental results}

Series of experiments on EAST aimed at characterizing the influence of ICRH on the SOL, by comparing the values of parameters measured by the reciprocating probes with and without ICRH, on L-mode plasma with currents of 450kA (Fig. 4). The probe was inserted two times per shot materialized by red and blue areas on Fig. 5, and remained deep in the SOL, behing the ICRF antenna limiter not to break the filament by plunging too deep. This explains why parameters measured are so small. Both ICRF antennas were also alternatively activated (Fig. 5), so that their respective influence can be compared. Figure 3 clearly shows that the K-port reciprocating probe was close and connected to the I-port antenna (red curves on Fig. 4), but far and not connected to the B-port antenna (blue curves on Fig. 4). Profiles in Fig. 4 show that ICRH significantly enhances potentials on connected regions where RF fields are more intense (red curve gets above the blue one as the probe approaches the plasma), presumably leading to ExB density convections consistent with pump-out effects observed (red curves). SOL rectifications in regions far and not connected to the active antenna are much smaller than in connected cases, yet those rectifications are significant as can be seen comparing blue (non-connected ICRF antenna) with dashed black curves (no ICRH).
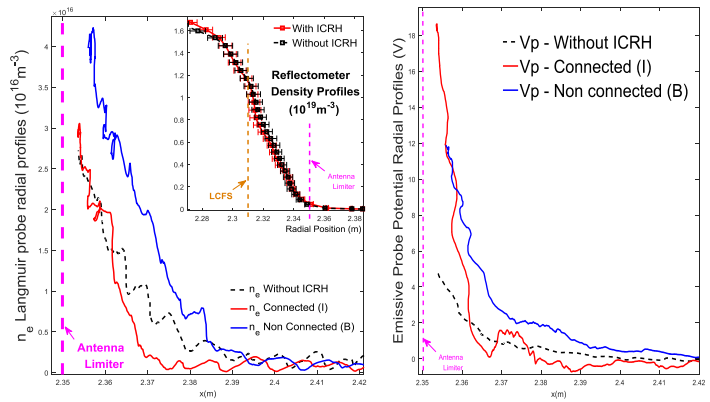

Fig. 4. Density and potential radial profiles respectively measured by Langmuir and emissive probes during reciprocations in the SOL in shots 64619 and 64623 . Black 
dashed curves represent cases without ICRH while red curves correspond to connected I-port antenna active and blue curves to non-connected B-port antenna active.

A density profile from the reflectometer was also added

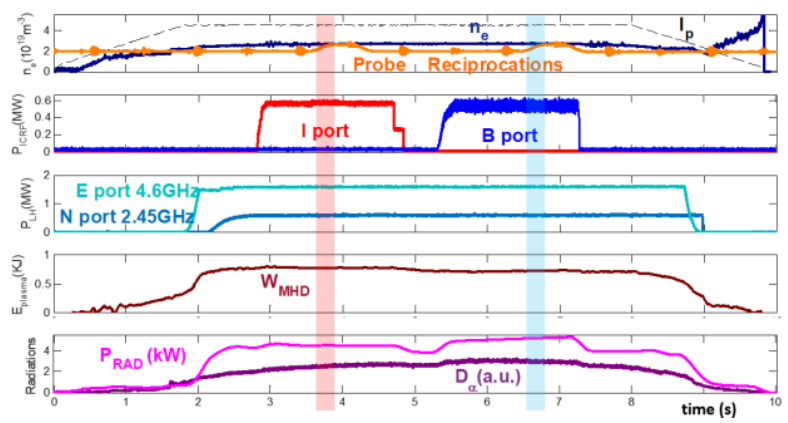

Fig. 5. Overview signals of the shot $\mathrm{N}^{\circ} 64619$ similar to 64623 . To the top are represented the line integrated density in blue, plasma current in dashed black and probe reciprocations in orange. Underneath we find each ICRF antenna power, then each LH grill power. Plasma energy is represented in in brown. Purple curve corresponds to plasma oscillations $\mathrm{D}_{\alpha}$ and radiations are in magenta.

During another series of experiments (Fig. 6), we tried to characterize the sheath formation at proximity and faraway of ICRF antennas for different power levels using a Langmuir reciprocating probe on the J-port and plunging until the LCFS. Fig. 7 shows floating potentials radial profiles measured for each reciprocation. Blue curves correspond to B-port launcher, which was launching relatively low power, was not magnetically connected and too far to significantly influence SOL parameters of the J-port. Red curves corresponding to Iport antenna launching much higher power and being very close and connected show much different trends. In the far SOL, oscillations between positive and negative values over one to two centimeters are characteristics of strong electric fields and convections. A typical point of the graph is the hump around the antenna limiter. This zone is subject to significantly higher potential gradients than others as it has been observed on most devices equipped with ICRH. Amplitude of oscillations also increases with ICRF power, indicating good correlation with RF sheath-induced mechanism.

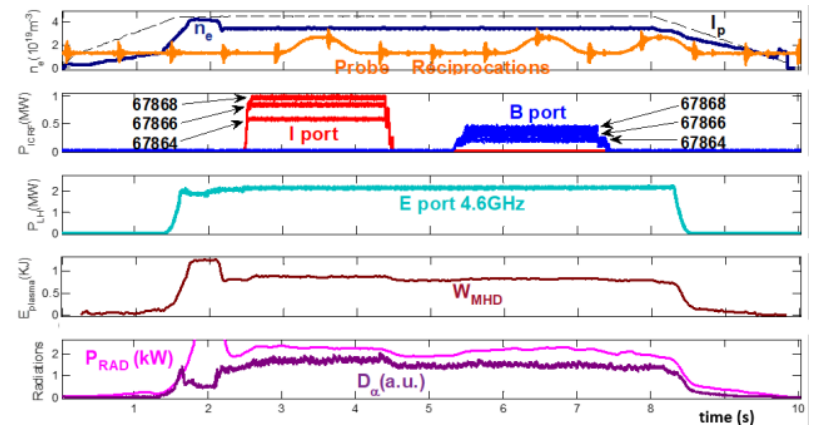

Fig. 6. Overview of the shots $\mathrm{N}^{\circ} 67864$ / 6 / 8 with respectively three level of ICRF power. Represented signals are the same as in figure 5.

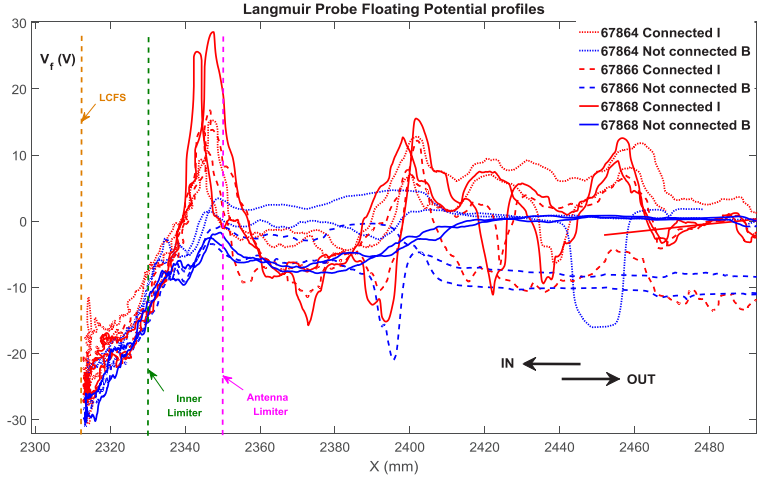

Fig. 7. Floating potential radial profiles measured by reciprocating Langmuir probe during shots $\mathrm{N}^{\circ} 67864$ (dotted), 67866 (dashed) and 67868 (full). Red curves (respectively

blue) correspond to I-port ICRF antenna active (B-port).

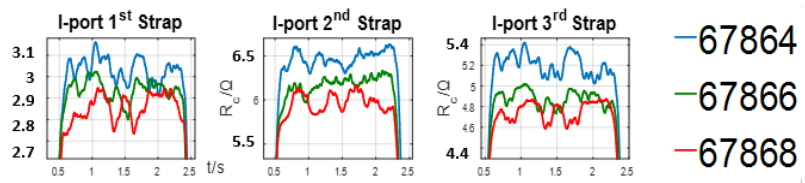

Fig. 8. Coupling resistances of I-port antenna current straps during shots $\mathrm{N}^{\circ} 6786467866$ and 67868 , showing that coupling efficiency decreases with power.

As a consequence, potential differences result in electric fields and ExB density convections. Also known as convective cells, those may locally increase evanescent layers width and have deleterious effect on the coupling efficiency of surrounding current straps as can be seen in Fig. 8.

In order to investigate even more the role of ICRF power, series of experiments with power modulations (Fig. 9) not only enabled to evidence the role of the power on near field sheath looking at ICRF coupling resistances (bottom right graph), but also on farther regions looking at LH reflection coefficients [7] (bottom left graph). ICRF-induced sheath basically enhances density convections in the SOL making waves coupling more difficult. Those observations remain true both in $\mathrm{L}$ and $\mathrm{H}$ modes.

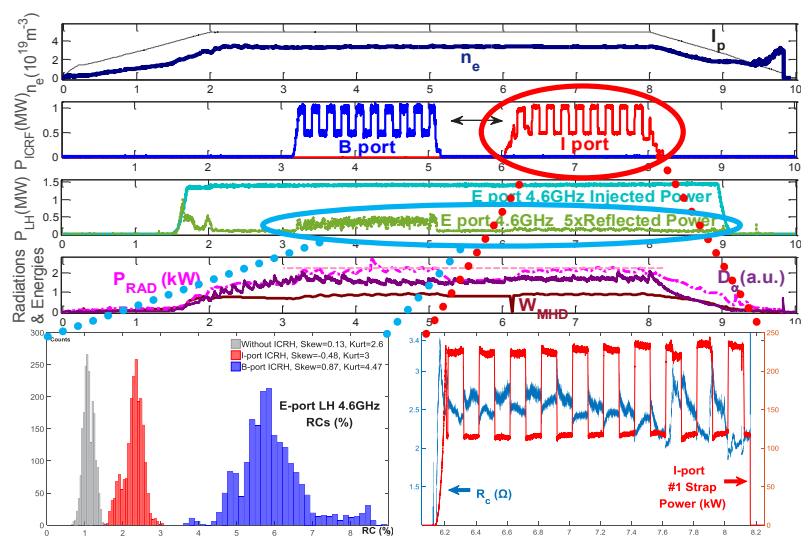

Fig. 9. Overview of the shot $\mathrm{N}^{\circ} 69949$ on top (same signals as in figure 5).

Bottom left bar chart shows reflection coefficients of the E-port 4.6GHz LH launcher at three different times, respectively without ICRH and both in non-connected and connected cases. Bottom right graph finally shows the coupling resistance of an I-port ICRF antenna current strap. All show deleterious impact of ICRH power.

While no influence of LHWs on ICRF was noticed in L-mode, on the contrary in H-mode, LHWs were found to have some positive effects $[8,4]$. Density gradient being stiff, in H-mode, 
pedestal tends to become sharper, density increases in the center more than at the edge and ICRF waves coupling becomes more complex. Fortunately, LHWs have been found to have a mitigating effect on ELMs [4] - changing type I into type III - diminishing their intensity and increasing their frequency. As can be seen in Fig. 10, this changes pedestal shape, it becomes softer, regulating edge densities, shortening ICRH waves evanescent length and improving the coupling. Such process could be very useful on ITER where it is planned to achieve high power H-mode plasmas.
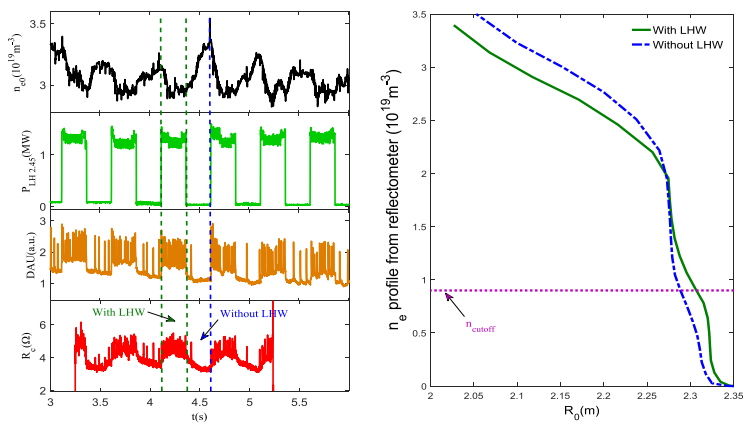

Fig. 10. Influence of LHWs on ICRF waves coupling in $\mathrm{H}-$ mode plasma. LH power (in green) mitigates ELMs (in orange), softening pedestal (right graph), decreasing core density at the benefit of the edge (black curve), improving ICRF waves coupling efficiency (coupling resistance in red)

\section{Conclusions and Prospects}

Over the EAST 2016 campaign, series of experiments in Lmode were carried out with the aim to characterize the mutual influence of ICRF and LH systems. In areas both magnetically connected and disconnected to active ICRF antennas, RF sheath was evidenced using a reciprocating probe allying both three tips Langmuir and emissive wire methods. No obvious influence was observed in non-connected regions, whereas in connected areas, SOL potential and temperature ( $\mathrm{cf}$ formulas in Appendix) tend to increase with RF power. Electric fields appear at the edge and seem to create density convections, consistent with both global density decrease and ICRF deleterious influence on LHWs coupling. Reciprocally, whereas no influence of LHWs on SOL parameters was observed in L-mode, they can mitigate ELMs in H-mode, which influences the plasma edge by softening the pedestal and improving ICRF coupling.

\section{Appendix}

We mentioned in the third part of this chapter that main advantages of the emissive probe are to provide a much more precise measurement of plasma potential, which can be written:

$$
\Phi=V_{E P}+\alpha_{h o t} \cdot T_{e} / e \approx V_{E P}+T_{e} / e
$$

with the hot probe correction coefficient:

$$
\alpha_{h o t}=\ln \left(\frac{I_{e s}}{I_{s}+I_{e m}}\right) \approx 1
$$

With $I_{e m}$ the emitted current, $I_{e s}$ and $I_{s}$ the electron and ion saturation currents respectively. Langmuir tips are in graphite; two of them are biased at $\pm 150 \mathrm{~V}$ and we assume it is sufficient to reach regimes of saturation in electrons $\left(\mathrm{V}_{\mathrm{p}}\right)$ and ions $\left(\mathrm{I}_{\mathrm{s}}\right)$. The third tip is measuring the floating potential $\mathrm{V}_{\mathrm{f}}$. Using the following formulas we calculate the electron temperature $T_{e}$ (equ 3 ), the electron density $n_{e}$ (equ 4 ) and the plasma potential $\Phi$ (equ 5); with e the electron charge, $\mathrm{m}_{\mathrm{i}}$ the ion mass and $\mathrm{A}_{\mathrm{pr}}$ the tip effective area:

$$
\begin{aligned}
& -T_{e}=\left(V_{p}-V_{f}\right) / \ln 2 \\
& -n_{e}=2 I_{s} /\left(e A_{\text {tip }} \sqrt{2 e T_{e} / m_{i}}\right) \\
& -\Phi=V_{f}+\alpha_{\text {cold }} \cdot T_{e} / e \approx V_{f}+2.8 T_{e} / e
\end{aligned}
$$

With the cold probe rectification coefficient

$$
\alpha_{\text {cold }}=\ln \left(I_{e s} / I_{s}\right) \approx \ln \left(\sqrt{\pi m_{i} / m_{e}}\right)=2.8
$$

Advantages of such triple probe are its cheap cost (simple electronic circuit), and very high time resolution, convenient for observing fast variation of plasma parameters due to turbulences. Nevertheless its sensitivity to RF sheath is its main drawback. The $\mathrm{RF} \rightarrow \mathrm{DC}$ rectification induced by ICRF waves shifts the I-V characteristic so that it is hard to determine if the fluctuations observed really reflect plasma dynamics or are just a mirage from DC rectification. For this reason, it is interesting to combine it with the emissive technique to benchmark the results with a better measure of the plasma potential (equ 5). For instance, combining relations 1 and 5 lead to another expression of the temperature, no longer depending on $\mathrm{V}_{\mathrm{p}}$ often leading to aberrant values due to electron drifts towards the edges in presence of RF E field - but only as a function of the floating potentials $\mathrm{V}_{\mathrm{f}}$ measured by the cold Langmuir probe and $\mathrm{V}_{\mathrm{EP}}$ measured by the hot emissive wire:

$$
T_{e}=\left(V_{E P}-V_{f}\right) /\left(\alpha_{\text {cold }}-\alpha_{\text {hot }}\right) \approx\left(V_{E P}-V_{f}\right) / 1.8
$$

\section{Acknowledgements}

The whole EAST ICRH team, colleagues in charge of reciprocating probes and French CEA colleagues; Laurent Colas and Annika Ekedahl are warmly acknowledged.

This work has been carried out within the framework of the EUROfusion Consortium and has received funding from the Euratom research and training program 2014-2018 under grant agreement No 633053. Views and opinions expressed herein do not necessarily reflect those of the European Commission. This work was supported partly by National key research and development program (grant nos 2016YFA0400600 and 2016YFA0400601). This work was supported partly by National Magnetic confinement Fusion Science Programme (grant nos 2015GB101001). This work was also supported partly by the National Natural Science Foundation of China under grant nos 11675213, 11375235 and 11375236.

\section{References}

1. L. Colas et al., PPCF 49 B35-B45 (2007)

2. R. Schrittwieser et al PPCF 44 567-578 (2002)

3. K.K Kirov et al PPCF 51044003 (2009)

4. A. Ekedahl et al, $26^{\text {th }}$ IAEA FEC 2016 (2016) EX/P7-34

5. Y. Peysson $20095^{\text {th }}$ Korean Fusion Plasma Summer School

6. E.H.Kong \& al. Plasma Physics and Controlled Fusion $\mathbf{5 4}$ (2012) 105003

7. Zhang Shoubiao et al 2014 Plasma Sci. and Technol. 16311

8. J. H. Zhang et al, 2017 Nucl. Fusion 57 (2017) 066030 